\title{
NYQUIST TRANSMISSION IMPROVED BY MULTIPLE TURBO CODES
}

\author{
YALAMARTHI BHARGAVI ${ }^{1} \&$ CH.RAVI KUMAR ${ }^{2}$ \\ ${ }^{1}$ Research scholar, Department of ECE, SIR. C.R.Reddy College of Engineering, \\ Eluru, Andhra University(AU), AP, India \\ Sr.Assistant Professor, Department of ECE, SIR C.R.Reddy College of Engineering ,
}

Eluru, Andhra Pradesh, India

Abstract
Working of the turbo code depends on their frame size but, the bit error rate (BER) action of turbo codes can
be improved by increasing the frame size. Miserably, the frame size increases produce some defects such as the increase
of transmission time and decoding delay. To overcome these drawbacks, we are using FTN scheme. In this scheme we
are using inter symbol interference (ISI), by using this method we achieved not only improves the BER performance but
also reduces the decoding delay and transmission speed but noise is little bit more so reduces of it we are using pre
coding method. By this method pre-equalizer is used. We consider the baseband system model for pre coded FTN
transmission scheme under an AWGN channel the system model is common for both linear and non-linear pre-
equalization methods. by this method we reduce the mainly noise and as well as increase the transmission speed and
decoding latency.
KEYWORDS: Turbo Codes, QAM Mapper, Pre Coded FTN, \& Pre Equalizer

Received: Oct 25, 2017; Accepted: Nov 28, 2017; Published: Dec 11, 2017; Paper Id.: IJECIERDDEC20172

\section{INTRODUCTION}

The context of error-correcting, coding, digital detection theory and explosion of wireless communication uses free interleaver, to solve the memory collision problem. To reduce the decoding latency, so using this FTN scheme the algorithms are the ones that improve the efficiency of the digital transmission in the radio part of a wireless network. These schemes have come to be faster than Nyquist signaling. The source is the measure of information is faster than Nyquist signaling. The source is the measure of information; basically source codes try to reduce the redundancy present in the source. Data compression is to minimize the average length of messages, faster than Nyquist (FTN) transmission scheme has been attracting more attention, as a spectral efficient transmission scheme. In the FTN transmission scheme, transmitted at a modulated symbols at a rate higher than the Nyquist transmission. Parallel to serial convolution is based on the analog to digital signal transformation for the decoding process, here decoding algorithm is used to max-log MAP

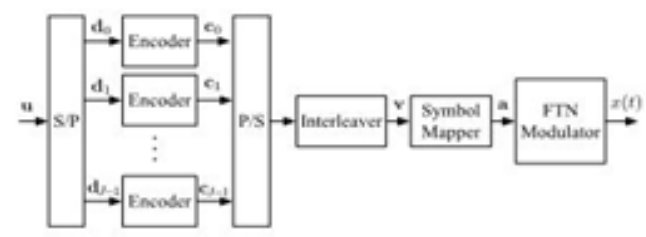

Figure1: Transmitter Block Diagram of Existed Scheme

Transmitter figure is FTN scheme is process to the structure. Basically, the information is transmitted through frames, so our information frame is $\mathrm{u}$ of size $\mathrm{K}$. The information frames is divided into subframes and the 
sub frames are encoded independently, by multiple turbo-like encoders before being presented at the FTN modulator. the information frame signal have in analogy signal that is converted to digital signal parallel to serial conversion is used to it. Symbol modulator; the symbol mapper takes consecutive bits and maps them to appropriate constellation points.

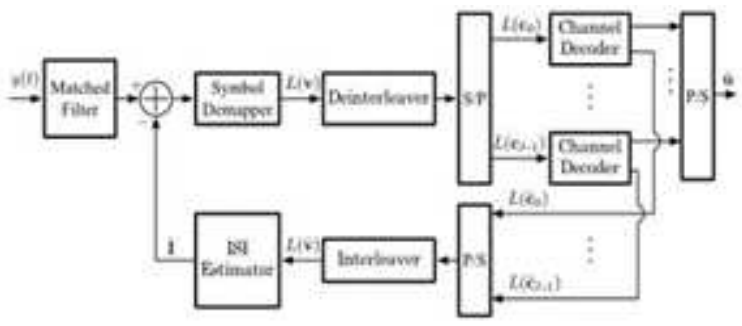

Figure 2: Receiver Block Diagram of the Existed Scheme

Multiplexed into a frame of size $K / r$ and then, inter leaved before being presented to the symbol mapper. The output of the inter leaver is given by

$$
\begin{gathered}
\mathrm{v}=\left\{v_{0}^{0}, v_{0}^{1}, \ldots, v_{0}^{m-1}, v_{1}^{0}, \ldots, v_{\frac{k}{r m}-1}^{m-1}\right\} \\
\quad \mathrm{P}\left\{c_{0}, c_{0}, \ldots, c_{j-1}\right\}
\end{gathered}
$$

Here, $\mathrm{P}\{$.$\} denotes the interleaving function and \mathrm{m}=\log _{2} M$ where $\mathrm{M}$ is a modulation order. The output of the interleaver $\mathrm{v}$ are mapped to $\mathrm{M}$-ary symbols, $\mathrm{S} \in\{s l \mid l=0,1, \cdots, M-1\}$ by an M-ary symbol mapper. The output of the symbol mapper a is given by

$$
a=\left\{a_{0}, a_{1}, \ldots, a_{\frac{k}{r m},-1}\right\}
$$

Here, $a_{n}=\mu\left(v_{n}^{0}, v_{n}^{1}, \ldots, v_{n}^{m-1}\right)$ where $\mu$ (.) denotes the symbol mapping function and $v_{n}^{k}, \mathrm{k}=0,1, \ldots, \mathrm{m}-$ 1 denotes the kth bit mapped to the M-ary symbol $\boldsymbol{a}_{\boldsymbol{n}}$. TheM-ary symbols a are presented to the FTN modulator and finally, the transmitted signal $\mathrm{x}(\mathrm{t})$ is given by

$$
x(t)=\sum_{n=0}^{\frac{k}{r m}-1} a_{n} h\left(t-n \tau T_{s}\right)
$$

where $\tau$ is an FTN factor, $\mathrm{h}(\mathrm{t})$ is the impulse response of a root cosine(RRC) filter and $1 / T_{s}$ is the Nyquist rate.

The block diagram of the proposed scheme, iterative joint demodulation and decoding is performed with in the receiver, i.e., the ISI induced by the FTN transmission is iteratively estimated and compensated based on the decoder output. The received signal y $(\mathrm{t})$ is matched filtered and sampled at every $\tau T_{s}$. The matched filter output samples $a_{n}$ are given by

$$
\widehat{a_{n}}=a_{n}\left(a_{n}+I_{n}\right)+N_{n}, \quad n=0,1, \ldots, \frac{k}{r m}-1
$$

Here, $\alpha n$, In and $N n$ denote an independent Rayleigh fading coefficient, the ISI due to the FTN modulator and an additive white Gaussian noise (AWGN) sample whose mean is zero and variance is N0/2 per dimension, respectively. In the symbol de mapper, log-likelihood ratios (LLRs) for $v_{n}^{k}$ are generated, based on the matched filter output samples, $a^{\wedge} n$ as follows. 


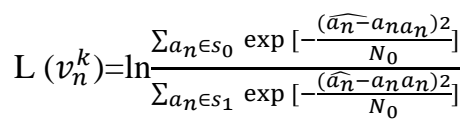

to understand the operation of the turbo decoder, let us begin with the definition of priori LLR (log-likelihood ratio), called a priori L-value, which is a soft value measuring how high the probability of a binary random variable $\mathrm{U}$ being +1 is in comparison with that of $U$ being -1 . This is a priori information known before the result caused by becomes available. While the sign of LLR

$$
\hat{u}=\operatorname{sign}\left\{L_{\mathbf{u}}(u)\right\}= \begin{cases}+1 & P_{\mathrm{u}}(u=+1)>P_{\mathrm{u}}(u=-1) \\ -1 & P_{\mathrm{u}}(u=+1)<P_{\mathrm{u}}(u=-1)\end{cases}
$$

is a hard value denoting whether or not the probability of $\mathbf{U}$ being +1 is higher than that of $\mathbf{U}$ being- 1 , the magnitude of LLR is a soft value describing the reliability of the decision based on $\widehat{U}$. Conversely, $p_{u}(u=+1)$ and $p_{u}(u=-1)$ can be derived from $L_{u}(u)$;

$$
P_{\mathrm{u}}(u)=\frac{e^{(u+1) L(u) / 2}}{1+e^{L(u)}}=\left\{\begin{array}{cc}
e^{L(u)} /\left(1+e^{L(u)}\right) & \text { for } u=+1 \\
1 /\left(1+e^{L(u)}\right) & \text { for } u=-1
\end{array}\right.
$$

Where $\mathbf{s} 0$ and $\mathbf{s} 1$ denote the set of $M$-ary symbols corresponding to $v_{n}^{k}=0$ and $v_{n}^{k}=1$,respectively. The LLRs generated at the de mapper are de-interleaved and then, de-multiplexed into $\mathrm{J}$ streams, before being presented to $\mathrm{J}$ identical channel decoding. Each channel decoder performs the first decoding iteration and generates LLRs for the information and the parity bits. The resulting LLRs are multiplexed and interleaved and then fed back to an ISI estimator. To estimate the ISI, soft symbol value $\widehat{a_{n}}$ is generated with the prior information on $v_{n}^{k}$, as follows:

$$
\begin{aligned}
\widehat{a_{n}}= & \sum_{l=0}^{M-1} s_{l} \mathrm{P}\left\{a_{n}=s_{l}\right\} \\
& =\sum_{l=0}^{M-1} s_{l} \prod_{k=0}^{m-1} P\left\{v_{n}^{k}=\mu_{k}^{-1}\left(s_{l}\right)\right\}
\end{aligned}
$$

Where $\mu_{k}^{-1}\left(s_{l}\right) \epsilon\{0,1\}$ denotes the kth bit mapped to $s_{l}$. Here, the a priori probabilities, $\mathrm{P}\left\{v_{n}^{k}=0\right\}$ and $\mathrm{P}$ $\left\{v_{n}^{k}=1\right\}$ are calculated based on the LLRs L $(\hat{v})$ generated with in the channel decoders as follows:

$$
\mathrm{P}\left\{v_{n}^{k}=b\right\}=\frac{\exp \left[(-1) b \frac{L\left(\widehat{v_{n}^{k}}\right)}{2}\right]}{\exp \left[+\frac{L\left(\widehat{v_{n}^{k}}\right)}{2}\right]+\exp \left[-\frac{L\left(\widehat{v_{n}^{k}}\right)}{2}\right]}, \mathrm{b} \in\{0,1\}
$$

and the ISI term $\widehat{I_{n}}$ can be estimated with the soft symbol values $\widehat{a_{n}}$ as follows:

$\widehat{I_{n}}=\sum_{k=0}^{\frac{k}{r m}-1} \widehat{a_{k}} \mathrm{~g}\left((\mathrm{n}-\mathrm{k}) \tau T_{s}\right)-\widehat{a_{n}}$

Here, $\mathrm{g}(\mathrm{t})=(\mathrm{h}(\mathrm{t}) * \mathrm{~h}(\mathrm{t}))$ where '*' denotes the convolution operator. Finally, the received symbol, $\widehat{a_{n}}$ are updated as follows:

$\widehat{a_{n}} \leftarrow \widehat{a_{n}}-a_{n} \widehat{I_{n}}$

And, the procedure described above is repeated for a pre deter- mined number of iterations. Modulator filter is connected to the summing point and divided to sub blocks symbol de-interleaved and ISI estimator interleaver serial to parallel converter is used, to estimated output data values. 
As it can be seen in Figure. 2, there are $J$ decoders and each decoders perform decoding operations for the received code frame of length $\frac{k}{r J}=\frac{N}{r}$ in parallel. Since the decoding latency is linearly depend on the length of code word, for a fixed information frame size $\mathrm{K}$, the decoding latency is reduced to $\frac{1}{J}$ in the proposed scheme compared to that of conventional coded FTN systems. Note that, for a fixed information frame size K, therequired decoding complexity is not depend on $\mathrm{J}$ and thus, the required decoding complexity of the proposed scheme is identical to that of the conventional coded FTN system.

Table 1: Parameters for the Turbo Code

\begin{tabular}{c|c}
\hline \hline Code rate, $r$ & $1 / 3,1 / 2$ \\
\hline Generating polynomials & $\left(1, g_{1} / g 2\right)=(1,5 / 7)_{8}$ \\
\hline Interleaver & QPP interleaver $[15]$ \\
\hline Interleaver length, $N$ & $128,256,512,1024,2048$ \\
\hline Decoving algonithm & Max-log MAP \\
\hline Channel models & AWGN, Rayleigh \\
\hline \hline
\end{tabular}

Here, this is the performance for the turbo code it is kind of all the requirements all placed over there different types of interleaver length. Channel models are using is AWGN, Rayleigh models these are so real able performance is given that channel.

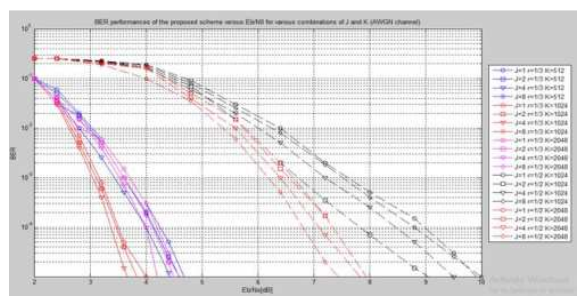

Figure.3: BER Performance of the Proposed Scheme Versus $\frac{E_{b}}{N_{0}}$ for Various

Combinations of $\mathbf{J}$ and $\mathrm{K}$ (AWGN Channel).

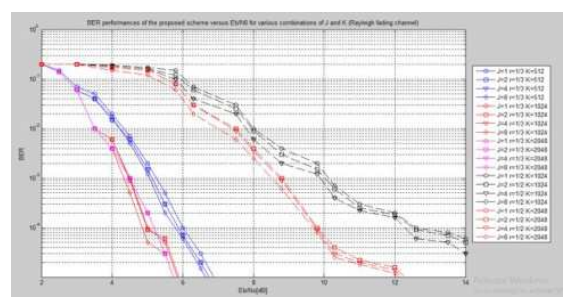

Figure.4: BER Performance of the Proposed Scheme Versus $\frac{E_{b}}{N_{0}}$ For Various Combinations of $\mathbf{J}$ and $\mathrm{K}$ (Rayleigh Fading Channel).

\section{PROPOSED SCHEME}

\section{PRECODED FTN}

We consider the baseband system model for precoded FTN transmission scheme under an AWGN channel; the system model is common for both linear and non-linear pre-equalization methods.

The data bits are first FEC encoded and then the interleaved and modulated data streama is precoded with a discrete-time pre-filter to produce 


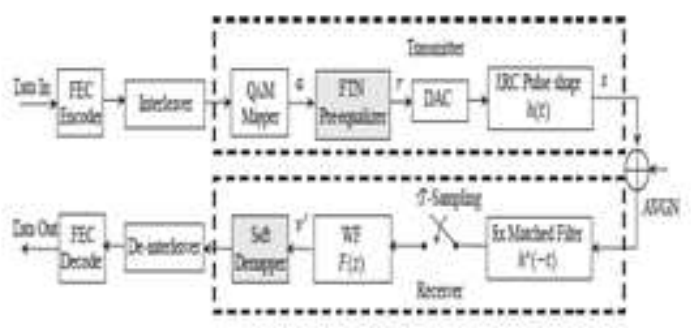

Figure.5: Proposed Scheme Block Diagram.

Baseband system model, for a pre-equalized FTN transmission, where the shaded blocks at the transmitter and the receiver represent the proposed FTN pre-equalizer and symbol de-mapper, respectively. The data symbols $r$. The preceded symbols $\mathrm{r}$ are pulse-shaped by a T-orthogonal pulse $\mathrm{h}$ and then, transmitted with an FTN acceleration factor _ <1. we assume a root-raised-cosine (RRC) Repulse-shaping filter $\mathrm{h}$ with a roll-off factor $\_$such that $1 \square 1 \mathrm{jh}(\mathrm{t}) \mathrm{j} 2 \mathrm{dt}=1$. At the receiver, the analog received signal, after passing through the matched-filter, is sampled at_T-intervals and then digitally processed by a noise whitening filter (WF) to whiten the collared noise due to FTN. Thereafter, the _T sampled signal v0 is sent to a symbol de-mapper to produce soft information in the form of LLRs for the FEC decoder.

The proposed scheme FEC it is forward error correction. In telecommunication, information theory and coding theory, FEC channel coding is a technique used for controlling errors in data transmission over unreliable or noise communication channels. QAM is quadrature amplitude modulation is both an analog and digital modulation scheme. It coverts two analog message signals, or two digital bit streams.

That process pre equalizer is used for filter to reduce the noise of signals.

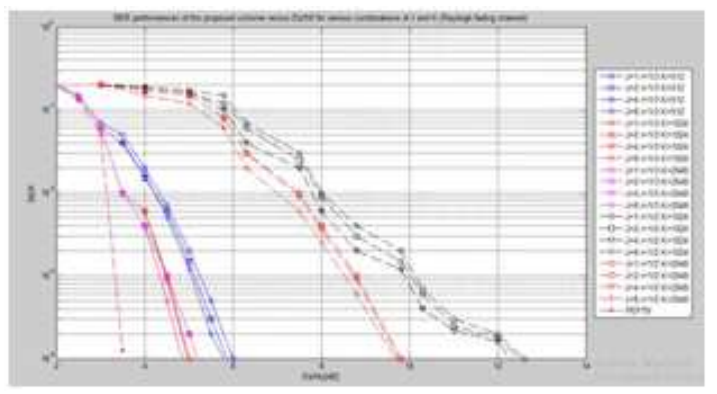

\section{Figure.6: BER Performance of the Proposed Scheme Versus $\frac{E_{b}}{N_{0}}$ For Various}

Combinations of $\mathbf{J}$ and $\mathrm{K}$ (AWGN Channel).

\section{NUMERICAL RESULTS}

For all numerical results the turbo code is used for channel code reliable results AWGN, Rayleigh fading channels are so prominent here $\mathrm{j}$ sub frames are used and then $E_{b}$ denotes the received energy per information bit. $\mathrm{k}$ is performance increases $\mathrm{j}$ is also increases. $\mathrm{k}$ is the code rate of $1 / 2$ and $1 / 3$. amount of performance is improved by the proposed scheme noise is also reduced to the signalling transformation transmission speed increases and delay of the decoding process is also reduced.

\section{CONCLUSIONS}

In this paper the FTN system which is at pre coded system to number of bit error rate performance is increases and also pre equalizer it acts as a filter, so the system noise may reduce to the proposed to be improved also signal 
transmission and delay time is also reduced information frame size is small then code rate is high. Proposed scheme is more prominent to the previous one and best signalling process is occurred.

\section{REFERENCES}

1. C.Berrouand

A.Glavieux, "Near optimum error

correcting

coding

anddecoding:Turbo-

codes,"IEEETrans.Commun.,vol.44,no.10, pp.1261-1271,Oct.1996.

2. R. G. Gallager, "Low-density parity-check codes," IEEETrans. Inf.Theory,vol.8,no.1,pp.21-28,Jan.1962.

3. D.Divsalar,H.Jin,andR.J.McEliece, “Codingtheoremsfor'turbo-

like'codes,"inProc.AllertonConf.Commun.,Control,Comput.,1998, pp.201-210.

4. S.BenedettoandG.Montorsi, “Unveilingturbocodes:Someresultsonparallelconcatenatedcodingschemes,”IEEETrans. Inf.Theory,vol.42, no.2,pp.409-428,Mar.1996.

5. R.Dobkin,M.Peleg,andR.Ginosar, "ParallelinterleaverdesignandVLSIarchitectureforlowlatencyMAPturbodecoders,"IEEETrans.VeryLarge ScaleIntegr. (VLSI) Syst., vol. 13, no. 4, pp. 427-438, Apr.2005.

6. C.-C.Wong,M.-W. Lai,C.-C.Lin,H.-C.Chang,andC.-Y.Lee, "Turbo decoder using contention-free interleaver and parallel architecture,"IEEEJ.Solid-StateCircuits, vol.45,no.2,pp.422-432,Feb.2010.

7. M. Katta Swamy, M. Deepthi, V. Mounika \& R. N. Saranya, Performance Analysis of DSSS and FHSS Techniques Over AWGN Channel, International Journal of Electronics, Communication \& Instrumentation Engineering Research and Development (IJECIERD), Volume 3, Issue 2, May - Jun 2013, pp. 7-14

8. J.E.Mazo, "Faster-than-Nyquist signaling,"BellSyst.Tech.J.,vol.54, no.8,pp.1451-1462,Oct.1975.

9. J.B.Anderson, F.Rusek, andV. Öwall, “Faster-than-Nyquistsignaling,”Proc.IEEE,vol.101,no.8,pp.1817-1830,Aug.2013.

10. Prlja, J. B. Anderson, andF. Rusek, "Receivers for faster-than- Nyquist signaling with and without turbo equalization,"inProc.IEEE Int.Symp.Inf.Theory,Jul.2008,pp.464-468.

11. A.J.Viterbi, "Error bounds for convolutional codes and an a symptoticallyoptimumdecodingalgorithm,"IEEETrans.Inf.Theory,vol.13, no.2,pp.260-269,Apr.1967.

12. L. Bahl, J. Cocke, F. Jelinek, andJ. Raviv, “Optimal decoding of linearcodesforminimizingsymbol errorrate,"IEEETrans. Inf.Theory, vol.20,no.2,pp.284-287,Mar.1974.

13. N.Benvenuto,R.Dinis,D.Falconer,andS.Tomasin, "Singlecarrier modulation with nonlinear frequency domain equalization: An idea whosetimehascome-Again," Proc.IEEE, vol.98,no.1,pp.69-96, Jan.2010.

14. F.RusekandJ.B.Anderson, "Multi stream faster than Nyquist signal- ing,”IEEETrans.Commun.,vol.57,no.5,pp.13291340,May2009.

15. P.Robertson, E.Villebrun,andP.Hoeher, "A comparison of optimal andsub-optimal MAPdecoding algorithms operating in the log domain,"inProc.IEEEInt.Conf.Commun.,Jun.1995,pp.1009-1013.

16. 3GPP,Technical Specification Group Radio Access Network;Evolved Universal Terrestrial Radio Access; Multiplexing and Channel Coding (Release8),3GPP, Sophia Antipolis, France,document36.212v8.4.0, Sep.2008. 